\title{
THE PROTOTYPE SOLID STATE INDUCTION MODULATOR FOR SLAC NLC
}

\author{
R.L. Cassel, J.E. DeLamare, M.N. Nguyen, G.C. Pappas, SLAC* \\ E. Cook, J. Sullivan, LLNA \\ C. Brooksby, Bechtel Nevada
}

\section{Abstract}

The Next Linear Collider accelerator proposal at SLAC requires a high efficiency, highly reliable, and low cost pulsed power modulator to drive the $\mathrm{X}$ band klystrons. The present NLC envisions a solid-state induction modulator design to drive up to 8 klystrons to $500 \mathrm{kV}$ for $3 \mu \mathrm{S}$ at 120 PPS with one modulator $(>1,000$ megawatt pulse, $500 \mathrm{~kW}$ average). A prototype modulator is presently under construction, which well power 4 each 5045 SLAC klystron to greater than $380 \mathrm{kV}$ for $3 \mu \mathrm{S}$ ( $>600$ megawatt pulse, $>300 \mathrm{~kW}$ Ave.). The modulator will be capable of driving the 8 each $\mathrm{X}$ band klystrons when they become available. The paper covers the design, construction, fabrication and preliminary testing of the prototype modulator.

\section{MODULATOR DESIGN CONSIDERATION}

The Next Linear Collider accelerator proposal at SLAC has selected the Solid State Induction Modulator approach for its X band klystrons because of its high efficiency, highly reliable, and low cost. The major difficulty with the conventional PFN type modulator use at SLAC for the Next Linear Collider (NLC) is the efficiency of the modulator for short pulse operation. The leakage inductance for the pulse transformer and the stray inductance of the switching circuit inherently limit the rise and fall time of the klystron voltage waveform. To reach the efficiency goals of $>75 \%$ for the modulator for the NLC it is necessary to have a rise and fall time of the klystron voltage pulse of less than $200 \mathrm{nsec}$. It is extremely difficult to obtain a fast rise time and high efficiency with a PFN modulator.

\section{THE PURPOSED INDUCTION MODULATOR FOR NLC}

The present modulator topology selected for the NLC modulator is similar to an induction accelerator. It consists of a large number of single turn induction cores each driven by its own solid-state switch. Due to the inherent low inductance of such a structure the secondary will have three turns. The resulting total leakage inductance at the secondary is extremely low ( $<20 \mu$ hy). The major part of the leakage induction is in the multiple primary side connections and drivers. The use of three turn secondary fractional turn transformer combined with two high current IGBT allows for the driving of 8 klystrons with one modulator or approximately 1000 megawatts of power for $3 \mu \mathrm{sec}$ see Figure 1.

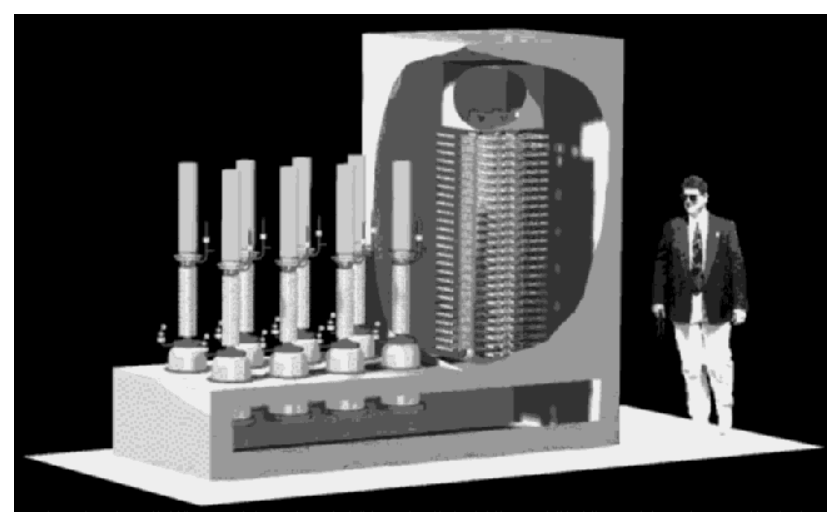

Figure 1. Induction Modulator Artistic Rendition

To obtain $500 \mathrm{kV}$ for $3 \mu \mathrm{sec}$ (1.5 volt seconds per turns) with a transformer, a three turns secondary required a large magnetic core cross sectional area. To drive the core without using a matched PFN requires a switch that can not only turn on fast at high power levels but also turn off. Switching devices now exist in the form of IGBT (Isolated Gate Bipolar Transistors). Figure 2.

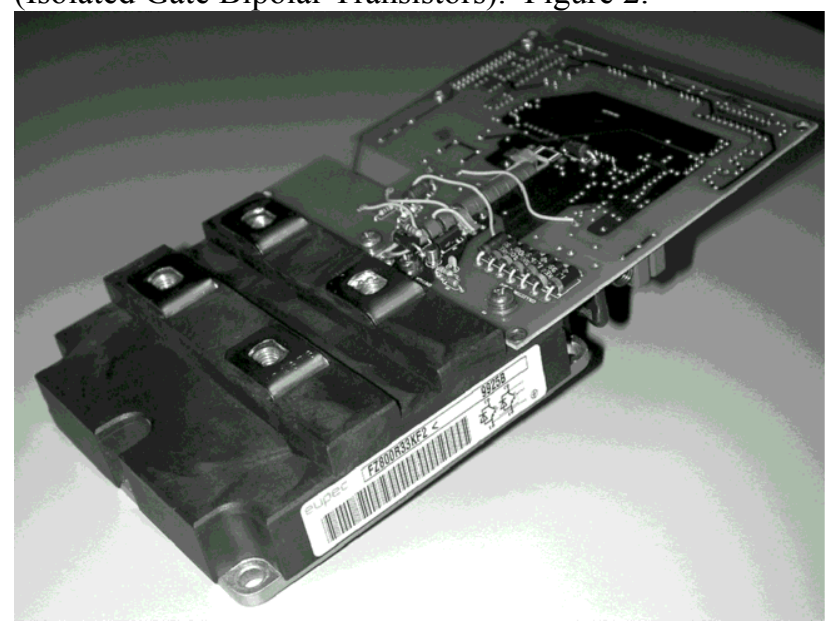

Figure 2. IGBT \& Gate Driver

\section{INDUCTION MODULATOR PROGRAM}

The NLC solid-state induction modulator R\&D program is divided into three Stages.

1) A Prototype 76 stack cores with three turns secondary to drive $500 \mathrm{kV}$ into water load and then $380 \mathrm{kV}$ into 4 each 5045 klystrons at full repetition rate, which is discussed in this paper.

2) A model using 10 cores and a standard pulse transformer to drive a SLAC 5045 klystron

3) A design for manufacturability prototype for 8 klystrons. 


\section{PROTOTYPE PROGRAM}

To obtain experience with the solid-state induction modulator at high voltage driving multiple klystron we are building a prototype modulator to drive 4 used SLAC 5045 Klystrons. (So called 4 dogs because they no longer provide acceptable RF output). Because the three-turn configuration is difficult to fabricate we are starting testing with a single turn into a water load without oil. We are measuring the rise time and total inductance of the stack, capacitive ringing compensation, and fault tolerance of the modulator.

\section{PROTOTYPE MODULATOR SPECIFICATION}

- Number of 5045 klystron

- Operating pulse voltage

- Operating pulsed current

- Repetition rate

- Rise /fall time

- Pulse duration flat top

- Energy Efficiency

- Number of secondary turns

- Number of fractional turns

- Number of core stacks

- Voltage per core

- Current per core

- Total leakage inductance

- Secondary stray capacitance

- Peak Power

- Average Power

\section{SOLID STATE DRIVE}

The core driver is simple, consisting of an IGBT, a DC charge capacitor in series with the IGBT driving the individual magnetic core. A capacitor with fast diode is used across the core to absorb the reflected energy from stray inductance under normal and fault conditions as well as the current if the one of IGBT is turned on later or off earlier then the other IGBTs. A pulse reset of the core is used to insure that the core is totally reset before the next pulse. The energy storage capacitor is charged through the transformer core. Figure 4.

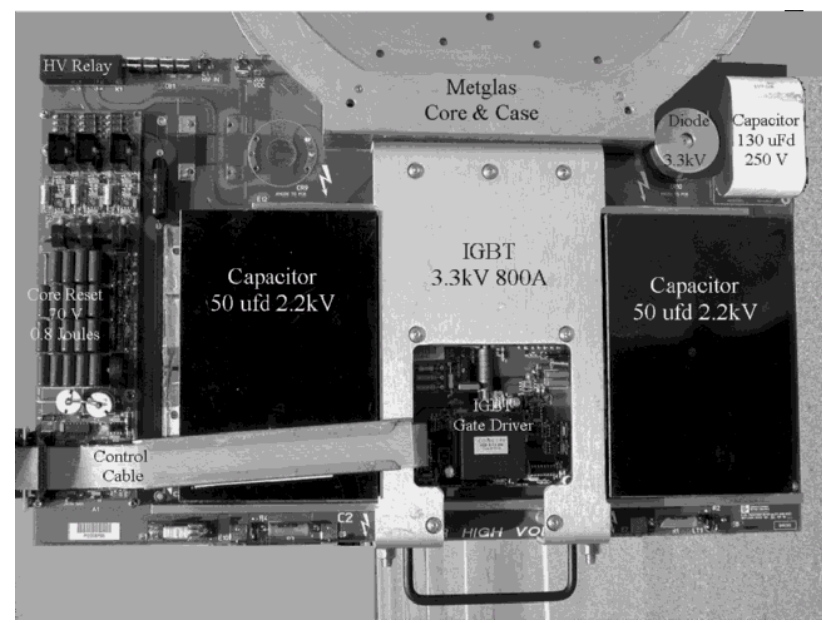

Figure 4. IGBT Drives boards

The core sections consist of two driver boards per core with one $3.3 \mathrm{kV} 800$ A IGBT per board. The driver boards are PC Board and arranged so that they can be plugged into the transformer core for easy replacement. Figure 5.

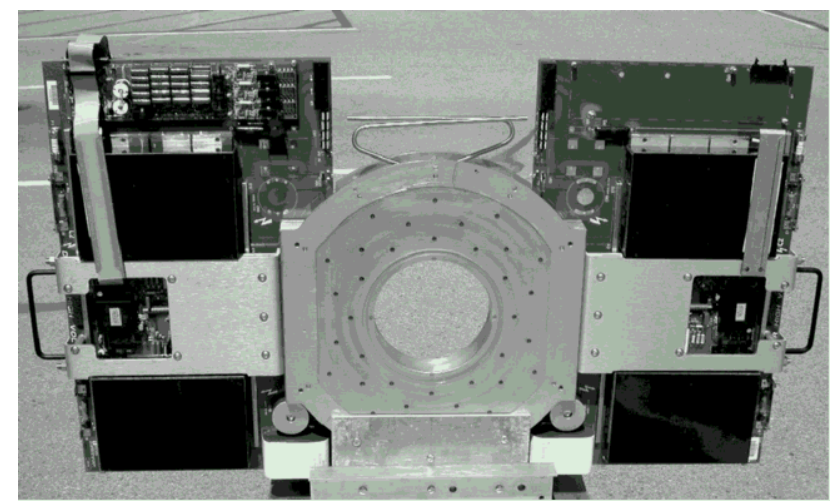

Figure 5. PC Board \&Core Driver

The core are stacked in two columns with the secondary threw the middle of the cores. An oil seal is made by orings on each core to allow for oil insulation of the secondary. The first test is with a single turn and no oil. The final stacked modulator is as shown in Figure 6.

The stacks are complete with half the drivers installed.

Figure 3. Metglas Core in case 


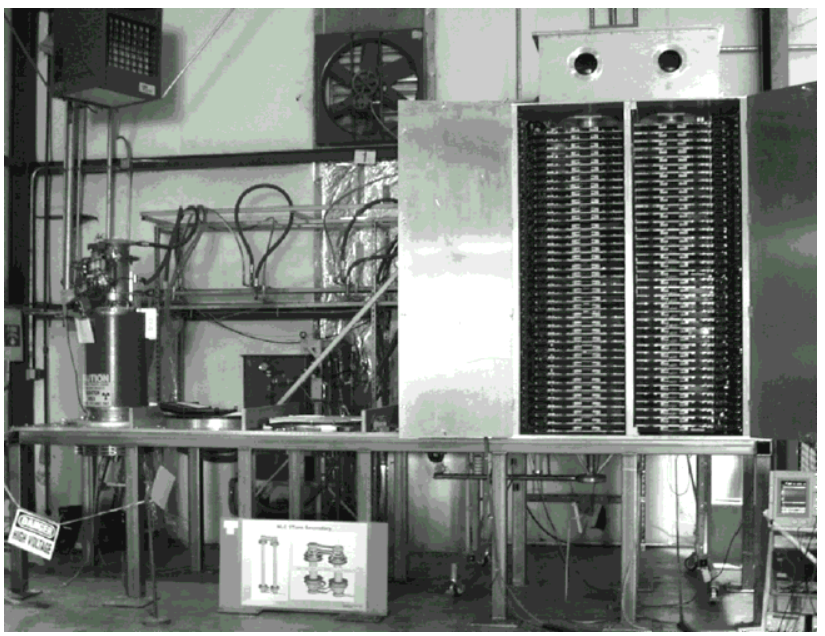

Figure 6. Assembled stack

The design of the three turns secondary is shown in figure 7 . The three turns are concentric coaxial structures with a stress level of less than $350 \mathrm{~V} / \mathrm{mil}$ in oil and $80 \mathrm{~V} / \mathrm{mil}$ on a insulator surface. Figure 7

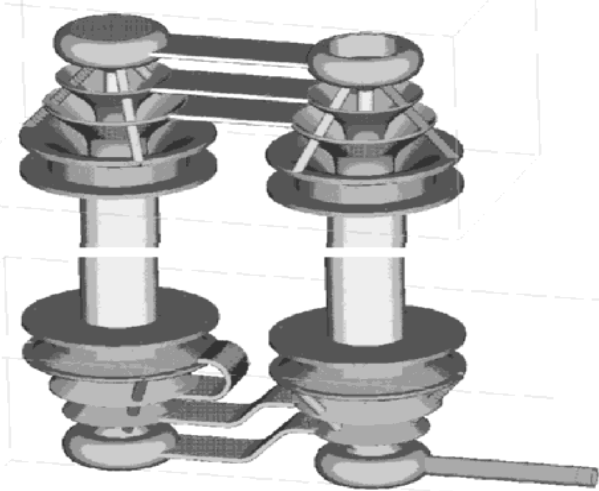

Figure 7. Three turn design

\section{PRELIMINARY TESTING}

With a single turn a total of $175 \mathrm{kV}$ pulse should be possible, but without oil the single turn and water load flashes over at proximally $80 \mathrm{kV}$. The waveform into a water load of $70 \mathrm{ohms}$ is shown in figure 8 .

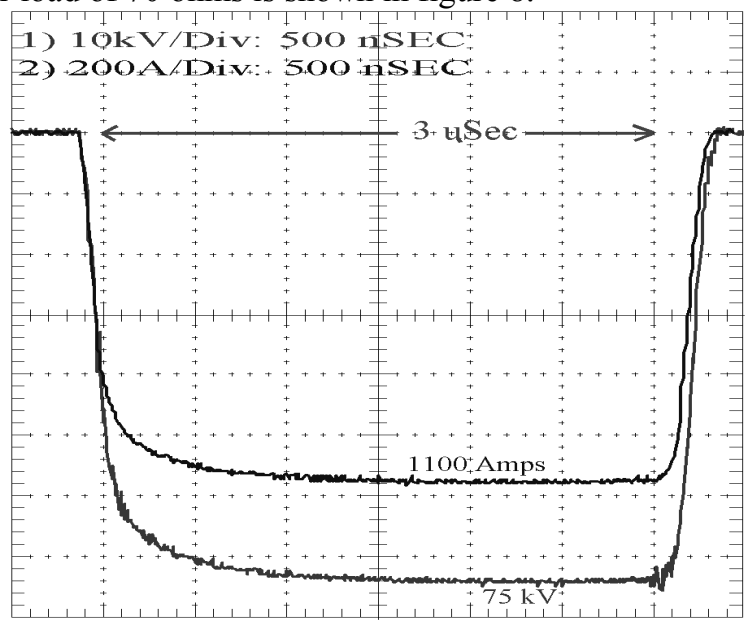

Figure 8. Voltage and current waveforms
The rounding on the rise time is actually an asset, because at this fast rise time the stray capacity of the klystron will cause ringing which is reduced by this waveform.

Spark down test where performed using both air and vacuum gaps to determent the fault clearing time of the modulator. Although the arc current is high the energy in the air both air and vacuum is less than 6 Joules due to the fast turn off time of the IGBTs. Figure 9

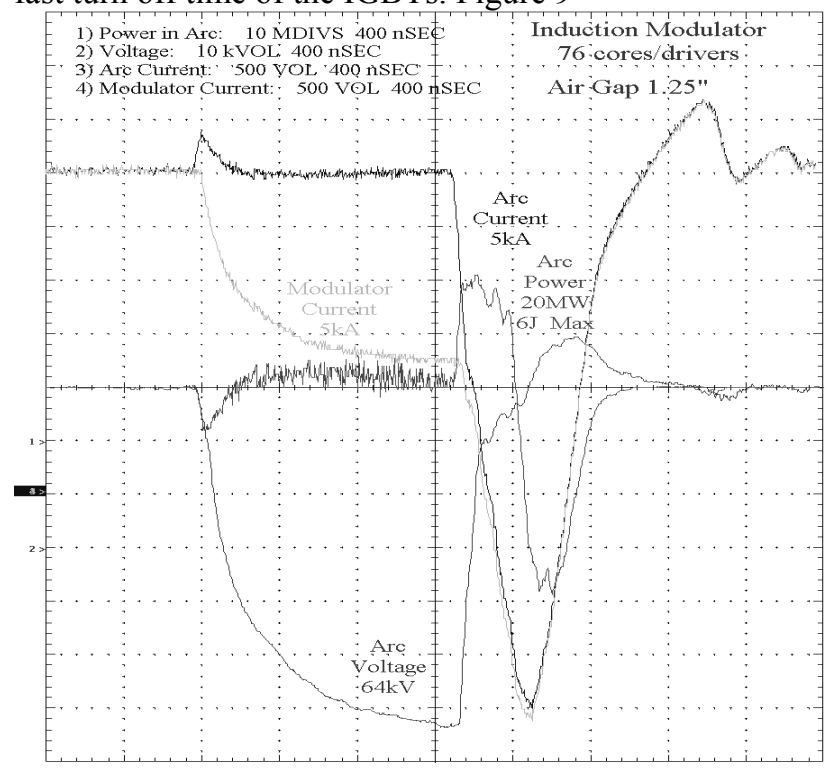

Figure 9. Arc over tests in air

\section{CONCLUSIONS}

The preliminary test on the prototype induction modulator indicate that the leakage inductances of each core including drivers is less than 30 nhy, and the single turn less than $2 \mu \mathrm{hy}$. The measured inductances of the stack are constantan with a total inductance of less than $20 \mu$ hy with three turns configuration. The pulsed waveforms have the rise time consistent with $<200 \mathrm{nsec}$ rise time and will ring with the klystron stray capacitance. The overshoot however can be corrected by timing adjustments of some of the IGBTs. The modulator is tolerant to arc faults and automatically turn off the pulse with very little energy going into the arc.

Further testing with the single turn are underway with the high voltage and power testing when the 3-turn secondary is ready.

\footnotetext{
"Work supported Department of Energy contract DE-AC03-76SF515
} 